\section{Determinantes do ganho ponderal excessivo durante a gestação em serviço público de pré-natal de baixo risco}

\section{Determinants of excessive weight gain during pregnancy in a public low risk antenatal care service}

Tamara E. Stulbach ${ }^{1}$

Maria Helena D'Aquino Benício'

Rosemarie Andreazza ${ }^{2}$

Silvia Kono'

'Depto de Nutrição, Faculdade de Saúde Pública, Universidade de São Paulo

2Universidade Federal de São Paulo

Correspondência: Tamara E. Stulbach. Rua Brasil, 70 apto 34. Centro. Guarujá, SP CEP 11410-320 E-mail: tamyst@ig.com.br

\section{Resumo}

Introdução: O excesso de ganho de peso durante a gestação pode ocasionar retenção de peso pós-parto e contribuir para a obesidade no sexo feminino. Metodologia: Neste estudo, avaliou-se a influência de fatores sociodemográficos, história gestacional, tabagismo, trabalho fora de casa e estado nutricional inicial sobre o ganho ponderal excessivo (GPE). O GPE foi estimado a partir das. recomendações do IOM (ganho semanal >0,58g, >0,53g e >0,39g, correspondentes a estado de nutricional no início da gestação: desnutrida, adequada e sobrepeso/ obesidade, respectivamente). Estudou-se uma coorte de 141 gestantes saudáveis, inscritas em serviço público de pré-natal, entre março de 1997 e março de 1998. A influência dos fatores de estudo sobre o GPE foi testada separadamente no $2^{\circ}$ e $3^{\circ}$ trimestres mediante análise de regressão de Poisson múltipla hierarquizada. Resultados: Dentre as 237 elegíveis houve 37,8\% de perdas, não se detectando diferenças estatisticamente significativas para as variáveis centrais do estudo. A incidência de GPE no $2^{\circ}$ trimestre foi de $38,6 \%\left(\mathrm{IC}_{95 \%} 30,5-47,2\right)$ e no $3^{\circ}$ trimestre foi de $36,4 \%\left(\mathrm{IC}_{95 \%} 28,5-45,0\right)$. No $2^{\circ}$ trimestre, apenas a escolaridade mostrou-se associada ao GPE. Em relação às mulheres com menos de 5 anos de escolaridade, as gestantes com 5 a 8 anos e acima de 8 anos de escolaridade apresentaram riscos relativos correspondentes a 2,09 $\left(\mathrm{IC}_{95 \%} 1,03-4,25\right)$ e 2,62 $\left(\mathrm{IC}_{95 \%} 1,32-5,22\right)$, respectivamente. No $3^{\circ}$ trimestre mostraram significância estatística as variáveis: escolaridade $>8$ anos $(\mathrm{RR}=1,91$ [ $\left.\mathrm{IC}_{95 \%} 1,22-2,97\right]$, ausência de companheiro $\left(\mathrm{RR}=1,66\right.$ [( $\left.\mathrm{IC}_{95 \%} 1,06-2,59\right]$, primiparidade $\left(\mathrm{RR}=2,13\right.$ [ $\left.\mathrm{IC}_{95 \%} 1,20-3,85\right]$ e estado nutricional inicial adequado e sobrepeso/obesi-

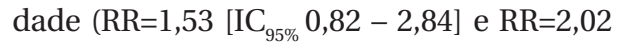
$\left[\mathrm{IC}_{95 \%} 1,04-3,92\right]$, respectivamente) em relação às desnutridas. Conclusão: Em função da elevada freqüência de GPE, particularmente em mulheres de escolaridade mais alta, as sem companheiro, as primíparas e aquelas com estado nutricional inicial adequado ou sobrepeso/obesidade durante a gestação, mais atenção deveria ser dada à prevenção e ao controle do problema durante o pré-natal.

Palavras-chave: Gestação. Ganho ponderal excessivo. IMC. 
Abstract

Introduction: Excessive weight gain during pregnancy tends to result in postpartum weight retention and contributes to obesity in women. Methods: This study evaluated the influence of socio-economic factors, obstetric history, smoking, having a job, and initial nutritional status on excessive weight gain (EWG). EWG was estimated according to IOM recommendations (weekly gain > $0.58 \mathrm{~g},>0.53 \mathrm{~g}$ and $>0.39 \mathrm{~g}$ which correspond to an initial nutritional status of underweight, eutrophic, overweight/obesity, respectively). We studied a cohort of 141 healthy pregnant women enrolled in public health services, between March 1997 and March 1998. The influence of each study variable on EWG was checked separately for the $2^{\text {nd }}$ and $3^{\text {rd }}$ trimesters using the Poisson model for hierarchy multiple regression analysis. Results: Amongst the 237 eligible pregnant women, there were $37.8 \%$ of patient losses, but no statistical significance was detected for the main study variables. The incidence of EWG during the $2^{\text {nd }}$ trimester was $38.6 \%\left(\mathrm{CI}_{95 \%} 30.5\right.$ -47.2 ) and during 3rd trimester it was $36.4 \%$ $\left(\mathrm{CI}_{95 \%} 28.5-45.0\right)$. During the $2^{\text {nd }}$ trimester only schooling was associated with EWG. When comparing women who studied less than 5 years, with the ones who studied 5 to 8 years and those with more than 8 years of schooling, relative risks were $2.09\left(\mathrm{CI}_{95 \%} 1.03\right.$ - 4.25) and $2.62\left(\mathrm{CI}_{95 \%} 1.32-5.22\right)$, respectively. In the $3^{\text {rd }}$ trimester the variables that resulted in statistical significance were: less than 8 years of schooling $\left(\mathrm{RR}=1.91\left[\mathrm{CI}_{95 \%}\right] .22\right.$ - 2.97], living without a partner $(\mathrm{RR}=1.66$ $\left[\left(\mathrm{CI}_{95 \%} 1.06-2.59\right]\right.$, primiparas $\left(\mathrm{RR}=2.13\left[\mathrm{CI}_{95 \%}\right.\right.$ 1.20 - 3.85]; adequate initial nutritional status and overweight/obesity $\left(\mathrm{RR}=1.53\left[\mathrm{CI}_{95 \%}\right.\right.$ $0.82-2.84]$ and $\mathrm{RR}=2.02\left[\mathrm{IC}_{95 \%} 1.04-3.92\right]$, respectively) in comparison to those who were underweight. Conclusion: Based on the high incidence of EWG, more attention should be given during antenatal care, particularly in women with more schooling, those living without partner, primiparas and those who have an initial adequate nutritional status or overweight/obesity during pregnancy, in order to prevent and control the problem.

Keywords: Pregnancy. Excessive weight gain. BMI.

\section{Introdução}

A avaliação antropométrica do estado nutricional durante a gestação é um procedimento de baixo custo, e de grande utilidade para o estabelecimento de intervenções precoces e eficazes durante a assistência pré-natal, com vistas à redução de riscos fetais e maternos. Na década de 90 , a Organização Mundial de Saúde (OMS) publicou uma extensa revisão acerca de aspectos metodológicos ligados à avaliação antropométrica durante a gestação, e o Institute of Medicine publicou um guia de recomendação de ganho de peso. Essas recomendações prevêem um aumento ponderal um pouco superior em relação às anteriores, encorajando as gestantes a aumentarem o consumo calórico ${ }^{1-7}$.

Estudo realizado com 120.531 gestantes de cinco estados norte-americanos participantes do programa "Special Suplemental Nutrition Program for Women, Infants and Children (WIC)", mostrou que a proporção de mulheres que ganharam peso dentro do recomendado diminuiu de $23,4 \%$ para $22,0 \%$, e a proporção de mulheres que ganharam peso acima do recomendado aumentou de $41,5 \%$ para $43,7 \%$, havendo portanto um aumento no ganho ponderal durante a gestação, entre os anos de 1990 e $1996^{8}$.

No Brasil, entre 1991 e 1995 foi realizado um estudo multicêntrico em seis capitais, abrangendo aproximadamente 3.082 gestantes clientes da rede pública de saúde. Menos de 1/3 das mulheres obteve ganhos de peso dentro da variação recomendada segundo o IOM. O percentual de ganho de peso acima do esperado $-52 \%$ - foi particularmente mais comum em mulheres que iniciaram a gestação acima do peso ${ }^{6,9}$.

A obesidade é atualmente um dos principais problemas de saúde pública, tanto em países desenvolvidos quanto em países em desenvolvimento ${ }^{10}$. Alguns estudos têm caracterizado o ganho ponderal excessivo durante a gestação como uma possível causa da obesidade entre as mulheres ${ }^{11-13}$.

O ganho excessivo durante a gestação, além de contribuir para a obesidade, está 
também associado a algumas complicações, entre elas a macrossomia fetal, as hemorragias, o trauma fetal, baixo peso ao nascer e mortalidade infantil ${ }^{9,13}$. Algumas características maternas estão associadas com o ganho de peso durante a gestação. Além dos fatores nutricionais (estado nutricional no início da gravidez e consumo energético), destacam-se os fatores sociodemográficos (escolaridade e idade), presença de companheiro, fatores obstétricos (paridade, intervalo interpartal,) e fatores comportamentais, como hábito de fumar e trabalho fora de casa ${ }^{6,14-16}$.

No Brasil, são escassos os estudos sobre os determinantes (fatores sociodemográficos, comportamentais e reprodutivos) do ganho ponderal excessivo durante a gravidez. Neste estudo, pretende-se avaliar a influência de algumas características maternas sobre o ganho ponderal excessivo durante a gestação, em gestantes clientes de um serviço público de pré-natal de baixo risco, no município de São Paulo.

\section{Métodos}

Estudou-se uma coorte de 141 gestantes saudáveis que iniciaram o acompanhamento de pré-natal no Hospital Maternidade Amparo Maternal, instituição filantrópica situada no Município de São Paulo, no período de março de 1997 a março de 1998. Participaram do estudo as gestantes com idade igual ou superior a 18 anos, e que apresentaram na primeira entrevista idade gestacional igual e/ou inferior a 18 semanas. Inicialmente, 260 gestantes ingressaram no estudo, das quais 227 foram consideradas elegíveis. As perdas foram de 37,8\% (86 gestantes), devido a abandono do prénatal (33), impossibilidade de obtenção da informação do peso ao nascer (43), mudança de cidade (8) e recusas (2).

As informações sobre a evolução da gestação atual, tabagismo, consumo de bebidas alcoólicas e dados socioeconômicos e de peso e altura foram obtidas durante as consultas de pré-natal, por intermédio de entrevistas (questionários pré-testados) e de exame antropométrico. As informações sobre a morbidade materna e dados do recém-nascido foram transcritos do prontuário clínico. As informações relativas aos bebês que nasceram em outros hospitais foram obtidas a partir do cartão da maternidade apresentado pela puérpera em visita domiciliar.

O peso da gestante foi coletado mensalmente durante a consulta de pré-natal, em balança digital eletrônica portátil "Soehnle", com capacidade máxima de 150 kg e precisão de 100 gramas. A estatura da mulher foi medida com estadiômetro portátil Seca com precisão de $0,1 \mathrm{~cm}$, coletada durante a primeira entrevista. As medidas antropométricas de peso e altura foram realizadas por antropometristas treinadas segundo técnica descrita em Lohman (1989) ${ }^{17}$ e padronizadas por metodologia proposta por Habicht $(1974)^{18}$.

O estado nutricional inicial das gestantes foi obtido através do Índice de Massa Corporal (IMC), calculado a partir da medida direta do peso obtido na primeira consulta de pré-natal (entre a $4^{\mathrm{a}}$ e a $18^{\mathrm{a}}$ semana) dividido pela altura ao quadrado. Para a classificação do estado nutricional inicial das mulheres em baixo peso, eutrofia, sobrepeso e obesidade, foram considerados os níveis críticos de Índice de Massa Corporal (IMC) para a idade gestacional propostos por Atalah et al. ${ }^{19}$ e adotados pelo Ministério da Saúde.

O ganho ponderal excessivo, variável resposta, foi obtido considerando-se o limite superior de ganho total de peso gestacional proposto pelo IOM $(1990)^{6}$ para cada categoria de estado nutricional.

O limite superior do ganho ponderal semanal no segundo e no terceiro trimestres de gestação foi estimado em duas etapas:

- subtração entre limite superior do ganho total e o ganho total relativo ao primeiro trimestre (até a $13^{\mathrm{a}}$ semana) de gestação.

- razão entre o resultado da $1^{\text {a }}$ etapa e duração do segundo e terceiro trimestres (27 semanas). 
Por exemplo, para gestantes com estado nutricional adequado: ((16 kg - 1,6 kg)/ 27 semanas) $=0,53 \mathrm{~kg} / \mathrm{semana}$.

Segundo o IOM $(1990)^{6}$, as recomendações de ganho de peso durante a gestação apresentam como limite superior de ganho ponderal semanal no segundo e terceiro trimestres os valores de 0,58g, 0,53g e 0,39g para gestantes com o estado nutricional inicial de baixo peso, eutrofia e sobrepeso/obesidade, respectivamente.

O peso ao nascer foi transcrito de informações da fornecidas pela maternidade (cartão da maternidade ou prontuário do recém-nascido). Classificou-se como baixo peso o recém-nascido com menos de $2.500 \mathrm{~g}$ ao nascimento. A idade gestacional foi avaliada pelo Método de Capurro ${ }^{18}$, sendo classificado como pré-termo o recém nascido com idade gestacional inferior a 37 semanas.

\section{Análise dos dados}

Para a detecção de eventuais diferenças entre os dois grupos (coorte de gestantes estudada e perdas de seguimento) adotou-se Teste do Qui-quadrado, com nível de significância de $5 \%$.

A influência dos fatores em estudo sobre o ganho de peso excessivo (GPE), foi inicialmente avaliada por análise univariada de regressão de Poisson com ajuste robusto da variância ${ }^{20}$. As variáveis que apresentaram associação estatisticamente significante com um $\mathrm{p}<0,20$ foram incluídas na análise de regressão múltipla, seguindo modelo hierarquizado preconizado por Victora et al. ${ }^{21}$.

No presente estudo, o modelo teórico hierarquizado dos fatores que influenciam o ganho ponderal excessivo no segundo e terceiro trimestres inclui como fatores mais distais os socioeconômicos (escolaridade materna, presença de companheiro e trabalho). Em seguida aparecem as variáveis relacionadas à história reprodutiva (idade, paridade e intervalo interpartal). As variáveis estado nutricional e hábito de fumar integram os blocos mais proximais.
A modelagem foi realizada em separado para o segundo e terceiro trimestres de gestação. Iniciou-se com as variáveis do bloco mais distal (socioeconômico) e a seguir foram introduzidos os blocos subseqüentes, um a um. Dentro de cada nível hierárquico foi considerado fator de risco para o ganho excessivo de peso as variáveis com $\mathrm{p}<0,05$, e fatores de ajuste para os blocos subseqüentes as variáveis com $\mathrm{p}<0,20$.

\section{Questões éticas}

O estudo foi realizado de acordo a portaria 196/96 do Ministério da Saúde e aprovado pelo Comitê de Ética da Faculdade de Saúde Pública/USP.

\section{Resultados}

A Tabela 1 mostra a comparação entre a coorte de gestantes estudada e as perdas de seguimento. Verifica-se que não há diferenças estatisticamente significativas entre os dois grupos para as variáveis centrais do estudo. Apenas o trabalho fora de casa foi mais freqüente nas perdas e o tabagismo tendeu a ser mais freqüente na coorte.

Com relação a coorte efetivamente estudada, verifica-se que cerca de um terço das gestantes situam-se na faixa de escolaridade de $5^{\mathrm{a}}$ a $8^{\mathrm{a}}$ série e outro terço na faixa de $2^{\circ}$ grau ou superior. A maioria das gestantes mora com seus companheiros e pertence à faixa etária entre 20 e 30 anos. Pouco mais da metade é primípara e nas multíparas predomina o intervalo interpartal superior a dois anos. Cerca de um quinto das mulheres fuma durante a gravidez. No início da gestação $21 \%$ das gestantes estavam desnutridas e $24 \%$ apresentavam sobrepeso ou obesidade segundo níveis críticos de IMC propostos por Atalah ${ }^{16}$.

A magnitude do ganho ponderal excessivo no segundo trimestre foi de $38,6 \%$ (IC $95 \% 30,5-47,2)$ e no terceiro trimestre de $36,4 \%$ (IC 95\% 28,5 - 45,0).

Verificou-se uma prevalência de $7,1 \%$ de baixo peso ao nascer. O peso médio ao 
Tabela 1 - Distribuição segundo as características socioeconômicas, história reprodutiva e outras características maternas das mulheres que integraram o estudo $(n=141)$ e das perdas de seguimento. São Paulo, março/1997 a outubro/1998.

Table 1 - Distribution according to socioeconomic status, reproductive history and other maternal characteristics of women in the study $(n=141)$ and loss to follow-up. São Paulo, March/1997 to

October/1998.

\begin{tabular}{|c|c|c|c|c|c|}
\hline \multirow[t]{2}{*}{ Variável/categorias } & \multicolumn{2}{|c|}{ Coorte } & \multicolumn{2}{|c|}{ Perda } & \multirow[t]{2}{*}{$\mathrm{p}$} \\
\hline & $\mathrm{N}$ & $\%$ & $\mathrm{~N}$ & $\%$ & \\
\hline \multicolumn{5}{|l|}{ Escolaridade (anos) } & 0,47 \\
\hline Até 4 a série & 41 & 30,6 & 23 & 28,8 & \\
\hline Da $5^{\mathrm{a}}$ a $8^{\mathrm{a}}$ série & 49 & 37,3 & 36 & 45,0 & \\
\hline $2^{\circ}$ grau ou superior & 43 & 32,1 & 21 & 26,3 & \\
\hline \multicolumn{5}{|l|}{ Mora com companheiro } & 0,64 \\
\hline Sim & 106 & 75,7 & 62 & 72,9 & \\
\hline Não & 34 & 24,3 & 23 & 27,1 & \\
\hline \multicolumn{5}{|l|}{ Paridade } & 0,93 \\
\hline Primíparas & 75 & 53,6 & 45 & 52,9 & \\
\hline Multíparas & 65 & 46,4 & 40 & 47,1 & \\
\hline \multicolumn{5}{|c|}{ Número de filhos nascidos vivos } & 0,52 \\
\hline Nenhum & 75 & 53,6 & 45 & 52,9 & \\
\hline 1 & 40 & 25,6 & 23 & 27,1 & \\
\hline 2 & 16 & 11,4 & 9 & 10,6 & \\
\hline 3 e mais & 9 & 6,4 & 8 & 9,4 & \\
\hline \multicolumn{5}{|l|}{ Intervalo interpartal (anos) } & 0,59 \\
\hline Menos de 1,5 & 11 & 15,9 & 4 & 9,8 & \\
\hline $1,5-2$ & 8 & 11,6 & 7 & 17,1 & \\
\hline 2 ou mais & 50 & 72,5 & 26 & 63,4 & \\
\hline \multicolumn{5}{|c|}{ Estado nutricional pré-gestacional } & 0,99 \\
\hline Desnutrição & 30 & 21,3 & 17 & 21,0 & \\
\hline Eutrofia & 77 & 54,6 & 44 & 54,3 & \\
\hline Sobrepeso/ Obesidade & 34 & 24,1 & 20 & 24,7 & \\
\hline \multicolumn{5}{|c|}{ Trabalho fora de casa no segundo trimestre } & 0,0003 \\
\hline Sim & 54 & 24,0 & 17 & 45,0 & \\
\hline Não & 71 & 76,0 & 21 & 55,0 & \\
\hline \multicolumn{5}{|c|}{ Trabalho fora de casa no terceiro trimestre } & 0,04 \\
\hline Sim & 36 & 31,0 & 16 & 50,0 & \\
\hline Não & 81 & 69,0 & 16 & 50,0 & \\
\hline \multicolumn{5}{|l|}{ Fumante } & 0,07 \\
\hline Sim & 29 & 22 & 11 & 12,8 & \\
\hline Não & 79 & 59,8 & 61 & 70,4 & \\
\hline Ex-fumante & 24 & 18,2 & 14 & 16,8 & \\
\hline
\end{tabular}

* Devido à presença de "missings" o total de casos analisado é inferior ao total de gestantes estudadas e perdidas no seguimento. "Due to the presence of "missings" total cases analyzed is below total pregnant women studied and loss to follow-up.

nascer foi de 3120g ( $\pm 497 \mathrm{~g}$ ), com idade gestacional média de 39 semanas $( \pm 1,3$ sem.). Quanto ao tipo de parto, verificouse uma freqüência de $30 \%$ de cesariana.

A influência das variáveis sociodemográficas, reprodutivas e de estilo de vida sobre o ganho ponderal excessivo no segundo e terceiro trimestres de gestação, de forma univariada, é apresentada na Tabela 1.

No segundo trimestre da gestação apenas a escolaridade materna exerce influencia estatisticamente significativa sobre 
o ganho ponderal excessivo - mais freqüente nos níveis mais altos de escolaridade. A ausência de companheiro eleva a magnitude do GPE com significância próxima ao nível critico de 0,05. Exceção feita ao trabalho fora de casa, as demais variáveis apresentaram significância estatística inferior a 0,20, o que as habilitou a entrar na análise múltipla como potenciais fatores de ajuste. O GPE foi mais freqüente nas primíparas, nas mulheres com intervalo interpartal maior que um ano e meio, idade inferior a 20 anos e superior a 30 anos, e nas tabagistas. Verifica-se também que em relação às desnutridas no início da gestação, as mulheres com estado nutricional adequado e as com sobrepeso/obesidade apresentaram elevação da incidência de GPE.

No terceiro trimestre de gestação apresentaram associação estatisticamente significativa com o GPE, a escolaridade materna, morar com o companheiro e paridade, sendo condições de risco a escolaridade mais alta, a primiparidade e a ausência de companheiro. O estado nutricional no início da gestação apresenta associação positiva com o GPE com $\mathrm{p}=0,20$ e por este motivo será incluído na análise múltipla. As demais variáveis apresentam associações ainda mais fracas com o GPE na mesma direção já descrita para o segundo trimestre de gestação.

Nas Tabelas 2 e 3 estão mostrados os resultados obtidos pela análise de regressão múltipla de Poisson com variância robusta.

Dentre as variáveis incluídas na análise múltipla referente ao segundo trimestre de gestação a escolaridade materna é a única que permanece no modelo. Considerando como nível basal as gestantes com escolaridade baixa (até $4^{\mathrm{a}}$ série), aquelas com escolaridade média ( $5^{\mathrm{a}}$ a $8^{\mathrm{a}}$ série) duplicaram o risco de ganho ponderal excessivo. Durante a modelagem as variáveis idade, morar com companheiro, paridade, intervalo interpartal, trabalhar fora de casa e o estado inicial não apresentaram p superior a 0,20 , e portanto não se mantive- ram no modelo nem como variáveis de ajuste.

Os resultados da análise realizadas para o $3^{\circ}$ trimestre mostram que as gestantes com nível de escolaridade alta $\left(2^{\circ}\right.$ grau/ superior) têm risco 1,9 vezes maior de obter ganho ponderal excessivo em relação às demais.

O risco de GPE também foi mais elevado para as gestantes sem companheiro $\left(\mathrm{RR}=1,7\left[\mathrm{IC}_{955}=1,06-2,59\right]\right)$ e para as primíparas $\left(\mathrm{RR}=2,13\left[\mathrm{IC}_{955}=1,20-3,85\right]\right)$. Com relação ao estado nutricional no início da gestação, as mulheres com peso adequado e as com sobrepeso/obesidade apresentam riscos 1,5 e 2 vezes mais elevados do que as desnutridas, respectivamente.

\section{Discussão}

Foi estudada uma coorte de gestantes saudáveis clientes de um serviço público de pré-natal de baixo risco do município de São Paulo que atende população carente do ponto de vista socioeconômico. Uma questão importante em estudo de coorte prospectivo, particularmente em populações urbanas de baixa renda, é a possibilidade de ocorrerem perdas durante o seguimento. Esta preocupação presente desde o início do estudo norteou a implementação de ações no sentido de minimizar as perdas. Mesmo assim, a magnitude das perdas de seguimento foi de $37,8 \%$; no entanto, a comparação com a coorte efetivamente estudada não detectou diferenças significativas para as variáveis centrais do estudo. É plausível supor que os resultados deste estudo possam ser aplicáveis a gestantes com as mesmas características daquelas que freqüentam serviços públicos de saúde de grandes centros urbanos do país.

No presente estudo, o estado nutricional no início da gestação foi avaliado por intermédio do IMC calculado a partir do peso e estatura medidos na primeira entrevista. O IMC foi categorizado 
Tabela 2 - Análise univariada entre as variáveis de estudo e o ganho excessivo de peso no segundo e terceiro trimestres de gestação.

Table 2 - Univariate analysis of study variables and excessive weight gain in the second and third pregnancy trimesters.

\begin{tabular}{|c|c|c|c|c|c|c|c|c|c|c|}
\hline \multirow[t]{2}{*}{ Variáveis/categorias } & \multicolumn{4}{|c|}{$\begin{array}{l}\text { Ganho ponderal excessivo } \\
\text { Segundo trimestre }\end{array}$} & \multicolumn{6}{|c|}{$\begin{array}{c}\text { Ganho ponderal excessivo } \\
\text { Terceiro trimestre }\end{array}$} \\
\hline & $\mathrm{N}$ & $\%$ & $\mathrm{RR}$ & IC ( 95\%) & $P$ & $\mathrm{~N}$ & $\%$ & $\mathrm{RR}$ & IC (95\%) & $P$ \\
\hline Escolaridade (anos) & & & & & $0,013^{*}$ & & & & & $0,007^{*}$ \\
\hline Até 4 a série & 41 & $19,5 \%$ & 1 & & & 41 & $31,7 \%$ & 1 & & \\
\hline a $5^{\mathrm{a}}$ a $8^{\mathrm{a}}$ série & 49 & $40,8 \%$ & 2,09 & $1,03-4,25$ & & 49 & $22,4 \%$ & 0,71 & $0,36-1,41$ & \\
\hline $2^{\circ}$ grau ou superior & 43 & $51,2 \%$ & 2,62 & $1,32-5,22$ & & 43 & $53,5 \%$ & 1,69 & $0,99-2,87$ & \\
\hline \multicolumn{11}{|l|}{ Escolaridade anos } \\
\hline Até $8^{a}$ série & - & - & - & - & & 90 & $26,7 \%$ & 2,01 & $1,29-3,12$ & 0,002 \\
\hline $2^{\circ}$ grau ou superior & - & - & - & - & & 43 & $53,5 \%$ & 1 & & \\
\hline Morar com companheiro & & & & & 0,10 & & & & & 0,018 \\
\hline Sim & 105 & $34,3 \%$ & 1 & & & 105 & $30,5 \%$ & 1 & & \\
\hline Não & 34 & $50,0 \%$ & 1,46 & $0,95-2,24$ & & 34 & $52,9 \%$ & 1,74 & $1,13-2,67$ & \\
\hline Paridade & & & & & 0,12 & & & & & $<0,001$ \\
\hline Primíparas & 75 & $44,0 \%$ & 1 & & & 75 & $50,7 \%$ & 1 & & \\
\hline Multíparas & 64 & $31,3 \%$ & 0,71 & $0,45-1,11$ & & 64 & $18,8 \%$ & 0,37 & $0,21-0,65$ & \\
\hline \multicolumn{11}{|l|}{ Intervalo interpartal (anos) } \\
\hline Menos de 1,5 & 11 & $9,1 \%$ & 1 & & 0,18 & 11 & $18,2 \%$ & 1 & & 0,93 \\
\hline $1,5-2$ & 8 & $37,5 \%$ & 4,12 & $0,51-33,26$ & & 8 & $25,0 \%$ & 1,38 & $0,24-7,90$ & \\
\hline 2 ou mais & 49 & $32,7 \%$ & 3,59 & $0,52-24,64$ & & 49 & $20,4 \%$ & 1,12 & $0,28-4,46$ & \\
\hline Idade materna & & & & & 0,171 & & & & & 0,63 \\
\hline$<20$ anos & 21 & $47,6 \%$ & 1,00 & & & 21 & $42,9 \%$ & 1,00 & & \\
\hline 20 a 30 & 96 & $33,3 \%$ & 0,70 & $0,41-1,19$ & & 96 & $35,4 \%$ & 0,83 & $0,40-1,72$ & \\
\hline 30 anos & 21 & $52,4 \%$ & 1,10 & $0,60-2,02$ & & 21 & $28,6 \%$ & 0,67 & $0,24-1,87$ & \\
\hline Trabalhar fora de casa & & & & & 0,87 & & & & & 0,47 \\
\hline Não & 71 & $36,6 \%$ & 1 & & & 81 & $32,1 \%$ & 1 & & \\
\hline Sim & 54 & $35,2 \%$ & 0,86 & $0,53-1,40$ & & 36 & $38,9 \%$ & 0,83 & $0,49-1,39$ & \\
\hline Fumante & & & & & $0,13^{*}$ & & & & & $0,22^{*}$ \\
\hline Sim & 29 & $31,0 \%$ & 1 & & & 29 & $27,6 \%$ & 1 & & \\
\hline Não & 79 & $38,0 \%$ & 1,22 & $0,66-2,26$ & & 79 & $39,2 \%$ & 1,42 & $0,74-2,72$ & 0,29 \\
\hline Ex fumante & 23 & $52,2 \%$ & 1,68 & $0,86-3,29$ & & 24 & $43,5 \%$ & 1,58 & $0,74-3,35$ & 0,24 \\
\hline Estado nutricional inicial & & & & & $0,32^{*}$ & & & & & \\
\hline Desnutrição & 30 & $30,0 \%$ & 1 & & & 30 & $26,7 \%$ & 1 & & $0,20^{*}$ \\
\hline Eutrofia & 77 & $40,3 \%$ & 1,34 & $0,73-2,48$ & & 77 & $37,7 \%$ & 1,41 & $0,73-2,74$ & 0,31 \\
\hline Sobrepeso/obesidade & 33 & $42,4 \%$ & 1,41 & $0,72-2,79$ & & 34 & $42,4 \%$ & 1,59 & $0,78-3,26$ & 0,20 \\
\hline
\end{tabular}

*Qui quadrado de tendência

Tendency chi-square

segundo os níveis críticos propostos por Atalah e adotados pelo Ministério da Saúde. Vale mencionar que estes níveis são bastante próximos dos recomendados pelo IOM.

Com relação ao ganho ponderal, verificou-se que cerca de $37 \%$ das 141 gestantes acompanhadas, apresentaram ganho de peso excessivo em relação ao recomendado pelo IOM/OMS para a categoria de estado nutricional inicial. Este resultado é discretamente superior ao encontrado em estudo já mencionado realizado em seis capitais brasileiras entre 1991 e 1995, com aproximadamente 3.082 gestantes, onde $29 \%$ das mulheres estudadas ganharam peso acima do recomendado ${ }^{9}$.

O estudo dos determinantes do ganho ponderal excessivo durante o segundo e terceiro trimestres de gestação, contem- 
Tabela 3 - Associação entre as variáveis sociodemográficas, reprodutivas e estilo de vida, e o ganho ponderal excessivo no segundo trimestre de gestação, mediante análise de regressão de Poisson múltipla hierarquizada.

Table 3 - Association between sociodemographic, reproductive and lifestyle variables, and excessive weight gain in the second trimester of pregnancy, through the Poisson model for hierarchy multiple regression analysis.

\begin{tabular}{|c|c|c|c|}
\hline Variáveis/categorias & Risco relativo ajustado & IC (95\%) & $\mathrm{P}$ \\
\hline Escolaridade ${ }^{1}$ & & & $0,003^{*}$ \\
\hline Até $4^{a}$ série & 1,0 & -—- & \\
\hline $5^{\mathrm{a}}$ a $8^{\mathrm{a}}$ série & 2,09 & $1,03-4,25$ & \\
\hline $2^{\circ} \mathrm{grau} / \mathrm{superior}$ & 2,62 & $1,32-5,22$ & \\
\hline
\end{tabular}

modelo 1 = escolaridade (A variável idade materna foi incluída de início devido ao $p=0,17$ na análise univariada. Na presença da escolaridade apresentou $\mathrm{p}=0,68$, não permanecendo como variável de ajuste. (Dados não apresentados)

* $=\mathrm{p}$ de tendência

model 1 = schooling (The variable maternal age was included from the beginning due to $p=0.17$ in univariate analysis. In the presence of schooling, $p=0.68$, and did not remain as an adjustment variable. (unavailable data)

$*$ trend $p$

plou de início uma análise de regressão de Poisson univariada.

A seguir foi realizada análise de regressão múltipla de Poisson hierarquizada. No segundo trimestre de gestação permane- ceram estatisticamente significantes apenas a variável escolaridade materna. Já no terceiro trimestre mantiveram-se estatisticamente significantes as variáveis escolaridade materna, morar com companhei-

Tabela 4 - Associação entre as variáveis sociodemográficas, reprodutivas e estilo de vida e o ganho ponderal excessivo no terceiro trimestre de gestação, mediante análise de regressão de Poisson múltipla hierarquizada.

Table 4 - Association between sociodemographic, reproductive and lifestyle variables, and excessive weight gain in the third pregnancy trimester, through the Poisson model for hierarchy multiple regression analysis.

\begin{tabular}{|c|c|c|c|}
\hline Variáveis/categorias & Risco relativo ajustado & IC (95\%) & $\mathrm{P}$ \\
\hline Escolaridade $^{1}$ & & & 0,005 \\
\hline até a $8^{a}$ série & 1,0 & -—-- & \\
\hline $2^{\circ} \mathrm{grau} /$ superior & 1,91 & $1,22-2,97$ & \\
\hline Morar com companheiro ${ }^{2}$ & & & 0,03 \\
\hline Sim & 1,0 & --- & \\
\hline Não & 1,66 & $1,06-2,59$ & \\
\hline Paridade $^{3}$ & & & 0,01 \\
\hline Multíparas & 1,0 & -— & \\
\hline Primíparas & 2,13 & $1,20-3,85$ & \\
\hline Estado nutricional inicial 4 & & & 0,029 * \\
\hline Desnutrição & 1,0 & $-\ldots$ & \\
\hline Eutróficas & 1,53 & $0,82-2,84$ & \\
\hline Sobrepeso/obesidade & 2,02 & $1,04-3,92$ & \\
\hline
\end{tabular}

Modelo 1= escolaridade ( $a$ idade não chegou a ser introduzida na modelagem pois apresentou um $p>0,20$ na análise univariada.) Modelo $2=$ modelo $1+$ morar com companheiro Modelo $3=$ modelo $2+$ paridade * $=\mathrm{p}$ de tendência

Model $1=$ schooling (age was not introduced in the modeling because it had a $p>0.20$ in the univariate analysis.) Model $2=$ model $1+$ living with partner Model $3=$ model $2+$ parity

* $=$ trend $p$ 
ro, paridade e estado nutricional inicial.

Gestantes com maior nível de escolaridade tenderam a ganhar mais peso no segundo e no terceiro trimestres de gestação. Resultado semelhante foi encontrado por $\mathrm{Kac}^{24}$ com relação à retenção de peso pós-parto em puérperas acompanhadas durante nove meses após o parto.

A variável morar com o companheiro apresentou associação estatisticamente significante com o GPE somente no terceiro trimestre de gestação. As gestantes que moram sozinhas têm risco $60 \%$ maior de apresentar GPE.

Em relação à paridade, observou-se que o risco de uma primípara ter ganho de peso excessivo no terceiro trimestre é duas vezes maior do que a de uma multípara . Resultado análogo foi encontrado no trabalho de Wolfe et al. ${ }^{23}$, realizado com 2,952 gestantes nos EUA, concluindo que gestantes primíparas brancas e negras obtiveram ganho ponderal excessivo durante a gestação, segundo as recomendações do IOM. O efeito da paridade sobre o ganho de peso e a adiposidade corporal foi discutido por Rodrigues ${ }^{24}$ em estudo transversal com 203 mulheres, no qual verificouse que aquelas que passam pela experiência da maternidade tendem a aumentar de peso.

Para o estado nutricional inicial observou-se neste estudo que as gestantes que iniciaram a gestação classificadas como eutróficas e sobrepeso/obesidade apre- sentaram risco de ganho excessivo de peso 1,5 e duas vezes maior do que as desnutridas no início da gestação. Nucci et al. ${ }^{9}$, encontraram em seu trabalho tendência semelhante.

Outros estudos com maior tamanho amostral são necessários para melhor avaliar os resultados aqui descritos e as relações entre o GPE e a idade materna, hábito de fumar, estado nutricional inicial, o trabalho e outras variáveis relacionadas ao estilo de vida durante a gravidez. Durante a última década, a prevalência de mulheres obesas tem crescido abruptamente, tanto em países desenvolvidos quanto em países em desenvolvimento, e o ganho de peso na gestação tem emergido como causa potencial do excesso de adiposidade. Segundo Gunderson et al. ${ }^{11}$ e Polley et al. ${ }^{25}$, o monitoramento da evolução ponderal e o aconselhamento nutricional são fundamentais para o controle adequado do ganho ponderal durante a gestação.

\section{Conclusão}

$\mathrm{Na}$ coorte estudada, a freqüência de GPE foi elevada no segundo e no terceiro trimestres da gestação. O problema apresenta maior magnitude entre as mulheres de maior escolaridade, e particularmente no terceiro trimestre naquelas sem companheiro, primíparas e com estado nutricional adequado ou sobrepeso/obesidade.

\section{Referências}

1. Feig DS \& Naylor CD. Eating for two: are guidelines for weight gain during pregnancy too liberal? The Lancet 1998; 351: 1054-5.

2. Naeye RL. Weight gain and the outcome of pregnancy. Am J Obstet Gynecol 1979; 135: 3-9.

3. Brown JE et al. Prenatal weight gains related to the birth of healthy - size infants to low - income women. JAm Diet Assoc 1986; 86: 1679-83.

4. Abrams BF \& Laros RK. Prepregnancy weight, weight gain and birth weight. Am J Obstet Gynecol 1986; 154: 503-9.

5. Brown JE. Weight gain during pregnancy what is “optimal”? Clin Nutr 1988; 7: 181-90.
6. [IOM] Institute of Medicine. Nutrition during pregnancy. Washington, D.C., National Academy Press, 1990. p. 2736 .

7. WHO Collaborative Study - Maternal anthropometry and pregnancy outcomes. Bulletin of the World Health Organization. 1995; 73(S): 1-98.

8. Schieve LA,Cogswell ME, Scanlon KS. Trends in pregnancy weight gain within and outside ranges recommended by the Institute of Medicine in a WIC population. Matern Child Health J 1998; 2(2): 111-6

9. Nucci L et al. Assessment of weight gain during pregnancy in general prenatal care services in Brazil. Cad Saúde Pública 2001; 17(6): 1367-74. 
10. Monteiro CA. Epidemiologia da Obesidade. In: Obesidade (Halpern A, Matos AFG, Suplicy HL, Macini MC \& Zanela MT ) 1999, Porto Alegre: Lemos Editorial, p15-30

11. Gunderson EP \& Abrams B. Epidemiology of gestational weight gain and body weight changes after pregnancy. Epidemiol Reviews 1999; 21:261-75

12. Scholl TO et al. Gestational weight gain, pregnancy outcome, and postpartum weight retention. Obstet Gynecol 1995; 86: 423-427.

13. Johnson JW \& Yancey MK. A critique of the new recommendations for weight gain in pregnancy. $A m J$ Obstet Gynecol 1996; 174(pt1): 254-8

14. Abrams BF \& Parker JD. Maternal weight gain in women with good pregnancy outcome. Obstet Gynecol 1990; 76(1): 1-7.

15. Dawes MG \& Grudzinskas JG. Patterns of maternal weight gain in pregnancy. British J of Obstet Gynaecology 1991; 98: 195-201.

16. Siega AM \& Adair LS. Biological determinants of pregnancy weight gain in a Filipino population. Am JClin Nutr 1993; 57: 365-72.

17. Lohamn et al. Anthropometric standardization reference manual. Human Kinetics Books. Champaign: Illinois; 1988.

18. Habicht JP. Estandarizacion de metodos epidemiologicos cuantitativos sobre el terreno. Bol Sanit Panam 1974; 117: 477-82.

19. Atalah ES, Castillo CL, Castro RS \& Áldea AP. Propuesta de un nuevo estándar de evaluación nutricional en embarazadas. Rev Méd Chile 1997; 125: 1429-36.
20. Puffer \& Serrano. Caracteristicas del peso al nascer. OPAS - Publicación Científica 504; 1987.

21. Capurro $\mathrm{H}$ et al. A simplified method for diagnosis of gestacional age in the newborn infant. JPediatr 1978; 93 (1): $120-2$.

22. Breslow NE. Generalizeed linear models: checking assumptions and strengthening conclusions. Statica Applicata 1996, 8: 23-41

23. Victora et al. The role of conceptual frame works in epidemiological analysis: a hierarchical approach. Int J Epidemiol 1997; 26: 224-7

24. Kac $\mathrm{G}$ et al. Fatores associados à obesidade abdominal em mulheres em idade reprodutiva. Rev Saúde Pública 2001; 35(1): 46-51

25. Wolfe WS et al. Parity-associated weight gain and its modification by sociodemographic and behavioral factors: a prospective analysis in US women. Int J Obesity 1997; $21: 802-10$

26. Rodrigues ML. Obesidade feminina: mudanças na adiposidade corporal após ciclo reprodutivo [dissertação de mestrado]. Brasília: Faculdade Ciência da Saúde da UNB, 1988.

27. Polley BA et al. Randomized controlled trial to prevent excessive weight gain in pregnant women. Int J Obesity 2002; 26: 1494-1502.

Recebido em: 07/10/05 Versão final reapresentada em: 03/08/06 aprovado em: 26/09/06 